\title{
Environmental Predictors of Zooplankton Biodiversity Across a Series of Polymictic Reservoirs
}

Alyssa Koshy, Kingsley lke-Anyanwu, Aaron Korbman, Michael Grove, Courtney Richmond, and Nathan Ruhl Department of Biological Sciences, Rowan University, Glassboro New Jersey, USA 08028

\section{Background}

- Polymictic reservoir series

- Beta-biodiversity: differences in species composition between sites.

- Zooplankton provides insight in seasonal succession of biodiversity for each ecosystem

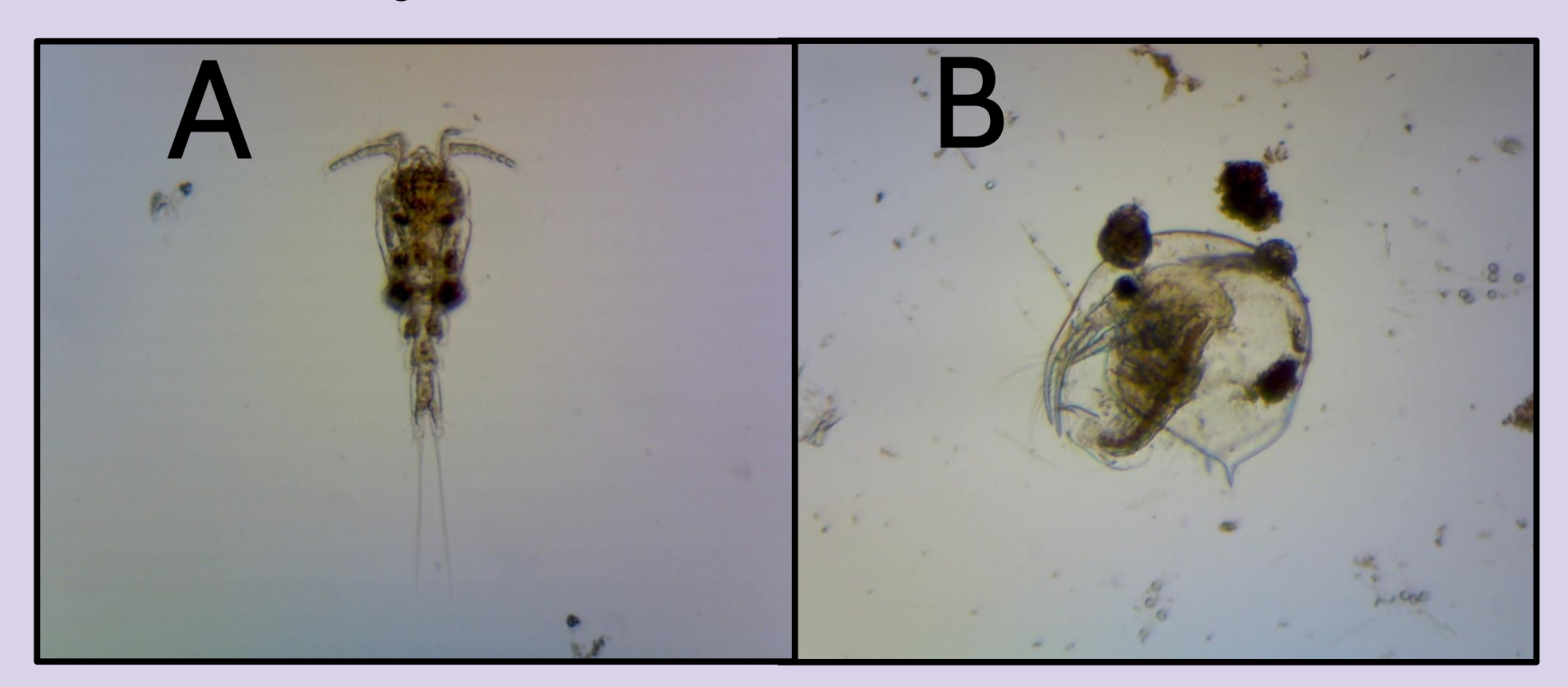

Figure 2: Copepod (A) and cladoceran $(B)$ at 40x magnification

Q1: Does zooplankton biodiversity differ across reservoirs connected in series? Table 1: A PERMANOVA with pairwise contrast for dam samples in 2016, 2017, and 2018

\begin{tabular}{|c|c|c|c|c|c|c|c|}
\hline \multicolumn{2}{|c|}{ Lake Pair } & \multicolumn{2}{|c|}{2016} & \multicolumn{2}{|c|}{2017} & \multicolumn{2}{|c|}{2018} \\
\hline Lake 1 & Lake 2 & $R^{\wedge} 2$ & P-value & $R^{\wedge} 2$ & P-value & $R^{\wedge} 2$ & P-value \\
\hline Elmer & Malaga & 0.16 & $\star * \star *$ & 0.212 & $\star * *$ & 0.186 & $* *$ \\
\hline Elmer & Parvin & 0.136 & $\star \star * *$ & 0.187 & *** & 0.178 & $* * *$ \\
\hline Elmer & Rainbow & 0.178 & $* * *$ & 0.218 & ** & 0.19 & $* * *$ \\
\hline Malaga & Parvin & 0.266 & & 0.055 & & 0.059 & \\
\hline Malaga & Rainbow & 0.035 & & 0.042 & & 0.037 & \\
\hline Parvin & Rainbow & 0.015 & & 0.007 & & 0.06 & \\
\hline Palatine & Malaga & $n / a$ & $n / a$ & 0.19 & $* *$ & 0.126 & \\
\hline Palatine & Parvin & $n / a$ & $n / a$ & 0.188 & $* * *$ & 0.09 & \\
\hline Palatine & Rainbow & $\mathrm{n} / \mathrm{a}$ & $n / a$ & 0.204 & *** & 0.115 & \\
\hline Palatine & Elmer & $n / a$ & $n / a$ & 0.085 & & 0.075 & \\
\hline
\end{tabular}

\section{Zooplankton Biodiversity 2016}

- Elmer different from Parvin, Rainbow and Malaga (Table 1)

- cHAB at Elmer

\section{Zooplankton Biodiversity 2017 and 2018}

- Elmer and Palatine different from Parvin, Rainbow and Malaga (Table 1)

- No cHAB in study system

We would like to thank PLVHA and the other research assistants for their help 